\title{
THE EFFECTS OF SEROTONIN INHIBITORS ON BONE METABOLISM: LITERATURE REVIEW
}

\author{
Rocco Franco ${ }^{1}$, Asaro Matte0 ${ }^{2}$, Francesco Gianfreda ${ }^{2}$, Michele Miranda ${ }^{2}$, Alberta Barlattani ${ }^{3}$, Patrizio Bollero ${ }^{2}$ \\ 'Department of Biomedicine and Prevention, University of Rome Tor Vergata, Rome, Italy \\ 2Department of Systems Medicine, University of Rome Tor Vergata, Rome, Italy \\ ${ }^{3}$ Department of Clinical Sciences and Translational Medicine, University of Rome Tor Vergata, Rome, Italy
}

\begin{abstract}
Depression is a mood and behavior disorder. It is a very frequent pathology in the world that requires pharmacological treatment using drugs that inhibit the recovery of serotonin. In fact, serotonin is a hormone that creates a feeling of well-being at the level of central nervous system. Serotonin is mainly produced by nerve cells. Therefore, selective serotonin reuptake inhibitors (SSRIs) are mainly used for the treatment of states of anxiety and major depressive disorder. SSRIs increase serotonin level, blocking its resorption in the presynaptic cell. Consequently, an increase in the bioavailability of serotonin in the synaptic junction occur. In fact, at the bone level, according to some observations, it causes an increase in bone resorption. Our study with a systematic review of the literature was to evaluate whether the intake of SSRIs could in any way influence implant survival. "SSRI" and "dental implant" were used as key words in the principal resources. Five articles that met the inclusion criteria were considered. The study found that these drugs can affect the implant survival rate. The data of these articles concerning implant survival have extrapolated. There is an association between intake and implant survival, probably due to a possible link between bone metabolism and drug. Further studies are recommended to effectively evaluate the influence of the drug on implant survival.
\end{abstract}

KEY WORDS: dental implant, selective serotonin reuptake inhibitor, implant success.

J Stoma 2020; 73, 3: 136-141

DOI: https://doi.org/10.5114/jos.2020.96942

\section{INTRODUCTION}

Depression is a social transformation that affects people's behavior and thinking process. It causes an alteration of person's sense of well-being and a change of relationships with others. The WHO has estimated that 350 million people are affected with depression. In the United States, antidepressant drugs are the third most prescribed class of drugs. Selective serotonin reuptake inhibitor (SSRI) belongs to the class of antidepressant drugs, which are frequently in use [1]. It is estimated that $60 \%$ of patients have been using these medications for about two years, while 14\% for about 10 years. The chronic intake of these drugs presents various side effects deriving from its use. In fact, in patients with a long and frequent intake, an increase in the risk of fracture and a decrease in bone density in both adolescents and adults were observed.

SSRIs have positive effects on mood. However, they have negative impacts on osteoblasts. The SSRI was the first class of antidepressant drugs discovered by scientists [2]. The mechanism of action of SSRI is to block the reuptake of serotonin to increase its concentration at the central level. All classes of SSRIs have a similar

\section{JOURNAL OF STOMATOLOGY CZASOPISMO STOMATOLOGICZNE}

ADDRESS FOR CORRESPONDENCE: Rocco Franco, PhD,

Department of Biomedicine and Prevention, University of Rome Tor Vergata, Rome, Italy, e-mail: roccofr91@gmail.com 
mechanism, i.e. blocking the reabsorption of serotonins and therefore, increasing their bioavailability. The drug has a high selectivity for serotonin receptors [3], but the interaction with other receptors causes various side effects. These drugs are generally well-tolerated and the common side effects are dose-dependent. The most frequent side effects are nausea and loose stool, headache, dizziness, somnolence or insomnia, sweating, tremor, dry mouth, anxiety, and restlessness. Less frequent side effects include weight gain, sexual dysfunction (inhibition of ejaculation or orgasm), bruxism, myoclonus, and paresthesia. Serotonin has several effects on bone metabolism; in fact, patients on this drug therapy have an annual decrease from $0.60 \%$ to $0.93 \%$ of the percentage of bone mineral mass. The molecular mechanism is not yet clear. However, serotonin acts on some receptors, including 5-HT1B, 5-HT2B, and 5-HT2C, and on the serotonin transporters (5-HTT), creating complex mechanisms at the level of osteoclasts and osteoblasts. These molecular mechanisms cause a negative effect on bone metabolism, increasing the differentiation of osteoclasts, and inhibiting the formation of osteoblasts. Dental implantology is the branch of dentistry that allows for a replacement of missing dental elements [4]. The osseointegration requires a strong and lasting connection between the bone and the implanted device, which is generally composed of titanium. These titanium devices are mainly used to replace missing teeth [5] and are an excellent therapeutic choice for restoring patient chewing. Failure to osseointegration can cause the possibility of reintervention by inserting a new device [6]. The good success of osseointegration is also given by the bone quality. In addition, SSRI has a negative effect on bone healing. Furthermore, it creates a difficulty in bone healing and decreases bone mineral density.

The purpose of this systematic literature review was to evaluate the possible effects of SSRI on implant osseointegration and implant survival.

\section{MATERIAL AND METHODS}

The study was conducted using three main scientific databases, including PubMed, Medline, and Web of Science. The timeframe considered for the electronic search was from March 1st, 2007 to March 1st, 2020. The term "SSRI" was first combined with "dental implant", and the web search was assisted with Mesh (medical subjects headings). The criteria for this review are described in PRISMA flow diagram. The purpose of this review was to find an answer to the following questions using PICO method (P: patient problem/population; I: intervention; C: comparison; O: outcome): Does the use of SSRIs increase the risk of implant failure and affect bone metabolism?

The PICO method aims to demonstrate whether the percentage of osseointegration in the group of pa- tients taking SSRIs is lower than in the healthy population. Therefore, the purpose of our study was to answer this question. The following inclusion criterion were used: articles in English, human studies, and clinical trials. Two independent people search with the same key words for all articles were used. The risk of bias during this phase was solved by an independent author that conducted the same search. The phase of screening was carried out by the two independent researchers that excluded an article duplication, reviews, and animal studies. There were 11 articles found in this phase. Three articles were excluded because are duplicates and they did not represent the topic proposed in this review. The phase of eligibility was conducted by other two reviewers. These authors compared the article founding and selected articles referred by PICO. Articles, which did not contain data regarding dental implant and SSRIs were excluded. At first, the authors were reading the abstract of all papers and 2 articles were eliminated, which did not respect the inclusion criteria. In this phase, the risk of bias was solved by an independent, completely external author, unknown to other authors. The number of articles remaining in this phase were 6. One article was excluded because did not use the periodontal index and described oral health only. The synthesis of data was carried out by the authors, with all data extracted. The author was firstly going through the abstract of all articles, after reading the complete test of the articles. All the reviewers extracted the data regarding the implant failure and the use of SSRIs. Articles, which did not contain the data and key words were excluded. All doubts regarding the included articles were solved by contacting the author (Figure 1).

\section{RESULTS}

Two independent scientists explored the previously mentioned key words, have read the titles, and summarized the abstracts of articles. During an initial reading, they excluded the articles that did not respect the topic. Therefore, articles that responded to the key characteristics were selected. The complete text of 5 remaining articles was read, and all were found to comply with inclusion criteria. In total, 6 articles were included in the present review. The scientists extrapolated data regarding implant failure rate in a population of patients taking SSRIs. Data comparing the rate of implant failure between patients taking SSRIs and a healthy control group is presented in Table 1. In a retrospective study, Wu et al. [7] included patients treated with dental implants from January 2007 to January 2013. In total, 916 dental implants of 490 patients were involved, of which 94 implants were inserted in 51 patients being treated with SSRIs. Furthermore, the risk of implant failure associated with the use of the drug was assessed in these patients. Also, other possible risk factors that could have influenced 

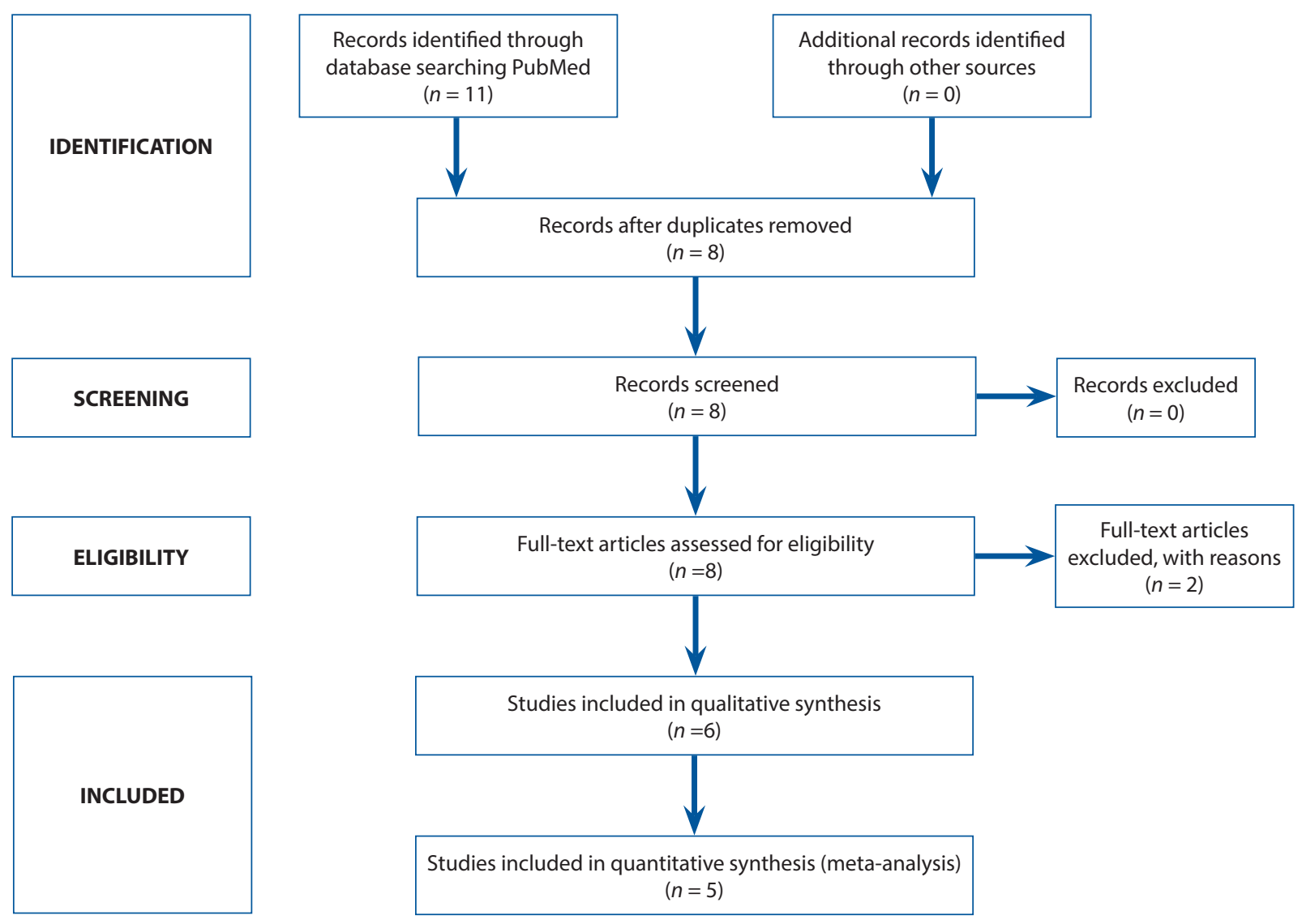

FIGURE 1. PRISMA flowchart

TABLE 1. Results of the review

\begin{tabular}{|c|c|c|c|c|c|c|}
\hline $\begin{array}{l}\text { Number } \\
\text { of implants }\end{array}$ & $\begin{array}{l}\text { Number of loose } \\
\text { implant }\end{array}$ & $\begin{array}{l}\text { Percentage } \\
\text { of implant failure }\end{array}$ & Author & $\begin{array}{c}\text { Observational } \\
\text { time }\end{array}$ & $\begin{array}{l}\text { Percentage of implant } \\
\text { failure in control group }\end{array}$ & $p$-value \\
\hline 94 & & 10.6 & Wu & $2007-2013$ & 4.6 & 0.003 \\
\hline 4,927 & 529 & & Carr & $1995-2014$ & & 0.006 (use of sertraline) \\
\hline 109 & 1 & 5.6 & Altay & 2 years & 1.85 & 0.166 \\
\hline 48 & $1980-2014$ & 12.5 & Charcanovic & & 3.3 & 0.007 \\
\hline 230 & $\begin{array}{c}27 \text { (diabetics), } \\
10.9 \text { (non-diabetics) }\end{array}$ & 27 & Deepa & & $\begin{array}{c}13 \text { (diabetics), } \\
4.1 \text { (non-diabetics) }\end{array}$ & \\
\hline
\end{tabular}

the measurement were analyzed. During 3 to 67 months of follow-up, in the control group, 38 of 784 implants failed, while 10 of 84 implants failed in the group of patients treated with SSRIs. Therefore, the data showed that the risk of implant failure was greater in patients treated with SSRIs (hazard ratio $[\mathrm{HR}]=6.28$; 95\% confidence interval $[\mathrm{CI}]: 1.25-31.61 ; p=0.03)$. The failure rate was $4.6 \%$ in the control group and $10.6 \%$ in the study group treated with SSRI. Furthermore, in the risk analysis, a close correlation between a narrow implant diameter and smoking habit with implant failure was assessed. Carr et al. [8] reported results of a retrospective study, in which patients receiving SSRI, who underwent im- plantation from January 1, 1995 to December 31, 2014 were analyzed. The history of using the drug and intake duration in the follow-up were evaluated. The associations between demographic characteristics and the use of SSRIs with implant failure were assessed with Cox proportional risk regression models, and the results were summarized with risk ratio (HR) and 95\% CI. Subsequent use of SSRIs was analyzed with time-dependent covariates. The total of 5,456 patients who received a dental implant with an average age of 53 years were analyzed. Follow-up of the 4,927 patients with no implant failure was 5.3 years. Implant failure for the remaining 529 patients occurred after 6 months. After evaluating possi- 
ble confounding factors including age, sex, and implant age, it was found that patients taking sertraline have an increased risk of implant failure $(\mathrm{HR}=1.60 ; 95 \% \mathrm{CI}$ : 1.15-2.23; $p=0.006$ ). Altay et al. [9] conducted a retrospective study with a total of 2,055 implants in 631 patients. 109 implants were inserted in 36 patients treated with SSRI, and 1,946 implants in 595 patients not treated for SSRI. This study aimed at whether SSRI intake was a negative factor for implant survival. The data was analyzed using Mann-Whitney test or Fisher's exact test accordingly. An average duration of follow-up for the group taking SSRIs was 21.5 months and 23 months for the control group ( $p=0.158)$. The failure rate for the SSRIs group was $5.6 \%$ and involved two patients. In contrast, the implant failure rate in the study group was $1.85 \%$ with eleven patients. After statistical analysis, the two groups showed no significant differences between SSRIs intake and implant survival $(p=0.166$ and $p=0.149$, respectively). The implant failure rate was 3,123 times higher in patients taking SSRIs than in the non-taking SSRIs study group. In particular, the group with SSRIs intake has a percentage of 3,005 times greater than an early implant failure. Charcanovic et al. [10] in a retrospective study evaluated the risk of implant failure in patients receiving SSRIs. In this work, patients taking SSRIs with no other systemic diseases or other confounding factors were included The implant success rate in patients with SSRIs was evaluated using different type of statistical analysis. However, the Fisher's test assessed the influence of SSRI on implant survival not considering possible confounding factors. In the SSRI group, via a multivariate generalized estimating equation, all possible local factors that could influence the result, such as the implant diameter, type of surgery, length of surface, and position were also analyzed. The author evaluated the parametric association between SSRI and implant failure considering all possible confounding factors. Therefore, 931 implants inserted in 300 patients were considered. There were 35 failures, with a percentage of $12.5 \%$ for patients taking SSRIs and 3.3\% for the control group, with Fisher's exact test $(p=0.007)$. The Fischer's test showed a statistically significant correlation but did not consider confounding factors. Multivariate GEE analysis indicated no association between SSRIs intake and implant failure. Deepa et al. [11] study evaluated the influence of SSRIs on implant success, and it was conducted on 352 patients with 680 dental implants registered. The clinical history and type of SSRI were recorded for each patient. Patients were divided into two groups: the first group included 110 patients treated with SSRIs, in whom 230 implants were placed, and the second group consisted of 242 patients, in whom 450 implants were placed and never used SSRIs. In each group, a follow-up was performed, and all implant failures were recorded. Group 1 included 35 patients $>50$ years of age, while 75 had $<50$ years of age. Group 2 included 60 patients aged $>50$, while 182 were $<50$ years old. Regarding implant failure, group 1 showed 25 failed implants, whereas group 2 demonstrated 21 failures. Thereafter, implant failures were differentiated by age groups. In group 1, patients over 50 years old showed 12 failures, while those under 50 years old experienced 13 failures. In group 2, there were 10 failures in the group of patients over 50 years of age and 11 failures in the group under 50 years of age. In the group 1, 27\% of diabetics had an implant failure compared to $13.4 \%$ of group 2 . This result was statistically significant $(p<0.05)$. The main failures found were in group 1 and involved screw loosening (8 patients), implant fracture (7 patients), periimplantitis (6 patients), and fracture screw (4 patients). In group 2, there were cases of loosening of lives (7 patients), fractures of screw (6 patients), fractures of implantation (5 patients), and peri-implantitis (3 patients). The difference was not statistically significant $(p>0.05)$ [11].

\section{DISCUSSION AND CONCLUSION}

Implant failure is an exceedingly rare event that occurs. There are many systemic diseases and conditions that increase the percentage of failure [12], such as diabetes, hypertension, and smoking. Currently, depression is a pathology frequently encountered, which can be caused by various problems associated with daily life. The main causes of implant failure are mainly peri-implantitis, mechanical overload, or a combination of these factors [13]. The early failures that often occur a few weeks or months after implant placement are due to implant contamination or lack of mechanical stability [14]. Failures that take place months or years after implant insertion are often due to peri-implantitis (progressive marginal bone loss induced by plaque), occurring mainly after a two-year follow-up, with implant loss due to mechanical overload at about 4-6 months [15]. Serotonin 5-hydroxytryptamine (5-HT) is a neurotransmitter of the monoamine family, which causes the feeling of well-being and happiness in the brain. Therefore, low serotonin levels can cause depression. A group of drugs widely used to treat depression are selective serotonin reuptake inhibitors (SSRIs), including celexa, paxil, lexapro, prozac, and zoloft. These drugs inhibit the recovery of serotonin and therefore, cause an increase in the neuronal level. Most patients with depression are treated with SSRIs [16]. Serotonin receptors are not only present in nervous cells, but also in many other tissues, for which the action of SSRIs has disappeared from side effects. Receptors can be found in the digestive tract, platelets, blood, and bones. Hence, side effects on these organs can occur due to SSRIs intake [17]. With regards to bone tissue, serotonin acts on bone tissue cells, specifically on 5-HT1B, 5-HT2B, 5-HT2C, and serotonin transporters (5-HTT). This interaction between receptors and bone tissue creates complex signals in osteoclasts and 
osteoblasts [18]. From in vitro studies, the serotonin reuptake receptor has been found to induce differentiation of osteoclasts. Animal studies have noted that fluoxetine has negative effects on both bone structure and density. In conclusion, SSRIs block the receptor 5-HTT on bone cells and has a direct negative effect on bone formation. It also increases the differentiation of osteoclasts causing a decrease in bone mass, increasing the differentiation of osteoclasts [19]. It has been observed that patients treated with SSRI have a decrease in bone density, with a rate of $0.60 \%$ to $0.93 \%$ [20-23]. Therefore, the purpose of various studies reviewed was to evaluate a possible interaction between SSRIs with implant survival. Various animal and in vitro studies have linked SSRI drugs to bone metabolism. In fact, in the studies analyzed, albeit few in the literature, most have found a higher percentage of implant failure compared to control groups [7-11]. Osteoclasts are stimulated to proliferate due to the presence of serotonin, whereas osteoblasts are inhibited to differentiate themselves from hematopoietic cells. SSRIs have a negative effect on mineral bone density and modifying trabecular cytoarchitecture. For this reason, a possible association between osseointegration and implant failure and SSRIs intake has been hypothesized [24-27]. Animal studies have shown that SSRIs have a negative effect on osteoblast differentiation in rats. Furthermore, it is interesting to note that SSRI drugs have significantly reduced the differentiation and expression of certain osteoblast genes that regulate bone turnover, including alkaline phosphatase, osterix, and osteocalcin [28, 29]. Carr's study specifically correlated which type of SSRIs has the greatest influence on implant survival [8] and demonstrated that sertraline has a greater influence on bone metabolism and therefore on implant failure. In addition, Carr et al. emphasized that implant failures occur mostly in patients who were taken SSRIs prior to implant treatment. This indicates that a long-term treatment can affect bone metabolism in a negative way. In Wu et al. [7] study, in accordance with Carr's report, the effects on implants arise mostly months after the implant surgery. The main cause of failure was implant overload. This means that SSRIs inhibit bone repair and load adaptation capabilities. All studies, except Altay's [9] and Charcanovic [10], revealed a statistically significant increase in the implant loss. However, in the Charcanovic study, there was a statistically significant correlation between implant loss and SSRsI intake, but the confounding factors were not taken into consideration. Therefore, all the analyzed studies are limited by the presence of multiple local factors that were not considered. However, main limitations of these studies were different. Firstly, the studies were designed as retrospectives, so the chance of data loss is extremely high. Secondly, none of the studies examined assess hygiene as a possible cause of an implant loss.

Furthermore, several studies considered in this review did not take into account possible confounding factors in the assessment of survival. It is now established that var- ious systemic pathologies or flawed habits can cause implant loss. Therefore, we can say that randomized studies will be needed to effectively evaluate the influence of SSRIs on implant survival. However, considering these data from the present review, we can conclude that these drugs have a negative effect on implant survival.

\section{CONFLICT OF INTEREST}

The authors declare no potential conflicts of interest with respect to the research, authorship, and/or publication of this article.

\section{References}

1. Beyer JL, Johnson KG. Advances in pharmacotherapy of late-life depression. Curr Psychiatry Rep 2018; 20: 34.

2. Aleksovski B, Neceva V, Vujović V, et al. SSRI-reduced platelet reactivity in non-responding patients with life-long recurrent depressive disorder: detection and involved mechanisms. Thromb Res 2018; 165: 24-32.

3. Joshi A. Selective serotonin re-uptake inhibitors: an overview. Psychiatr Danub 2018; 30: 605-609.

4. Lochmann D, Richardson T. Selective serotonin reuptake inhibitors. Handb Exp Pharmacol 2019; 250: 135-144.

5. Owens MJ. Selectivity of antidepressants: from the monoamine hypothesis of depression to the SSRI revolution and beyond. J Clin Psychiatry 2004; 65 Suppl 4: 5-10.

6. Son SE, Kirchner JT. Depression in children and adolescents. Am Fam Physician 2000; 62: 2297-2312.

7. Wu X, Al-Abedalla K, Rastikerdar E, et al. Selective serotonin reuptake inhibitors and the risk of osseointegrated implant failure: a cohort study. J Dent Res 2014; 93: 1054-1061.

8. Carr AB, Gonzalez RLV, Jia L, Lohse CM. Relationship between selective serotonin reuptake inhibitors and risk of dental implant failure. J Prosthodont 2019; 28: 252-257.

9. Altay MA, Sindel A, Özalp Ö, et al. Does the intake of selective serotonin reuptake inhibitors negatively affect dental implant osseointegration? A retrospective study. J Oral Implantol 2018; 44: 260-265.

10. Chrcanovic BR, Kisch J, Albrektsson T, Wennerberg A. Is the intake of selective serotonin reuptake inhibitors associated with an increased risk of dental implant failure? Int J Oral Maxillofac Surg 2017; 46: 782-788.

11. Deepa, Mujawar K, Dhillon K, Jadhav P, Das I, Singla YK. Prognostic implication of selective serotonin reuptake inhibitors in osseointegration of dental implants: a 5-year retrospective study. J Contemp Dent Pract 2018; 19: 842-846.

12. Esposito M, Hirsch JM, Lekholm U, Thomsen P. Biological factors contributing to failures of osseointegrated oral implants (II) etiopathogenesis. Eur J Oral Sci 1998; 106: 721-764.

13. Tonetti MS, Pini-Prato G, Cortellini P. Effect of cigarette smoking on periodontal healing following GTR in infrabony defects. J Clin Periodontol 1995; 22: 229-234.

14. Verdel BM, Souverein PC, Egberts TC, Van Staa TP, Leufkens HG, de Vries F. Use of antidepressant drugs and risk of osteoporotic and nonosteoporotic fractures. Bone 2010; 47: 604-609.

15. Wong M, Eulenberger J, Schenk R, Hunziker E. Effect of surface topology on the osseointegration of implant materials in trabecular bone. J Biomed Mater Res 1995; 29: 1567-1575.

16. Battaglino R, Fu J, Späte U, et al. Serotonin regulates osteoclast differentiation through its transporter. J Bone Miner Res 2004; 19: 1420-1431. 
17. Diem SJ, Blackwell TL, Stone KL, et al. Use of antidepressants and rates of hip bone loss in older women: the study of osteoporotic fractures. Arch Intern Med 2007; 167: 1240-1245.

18. Eom CS, Lee HK, Ye S, Park SM, Cho KH. Use of selective serotonin reuptake inhibitors and risk of fracture: a systematic review and meta-analysis. J Bone Miner Res 2012; 27: 1186-1195.

19. Rosa A, Miranda M, Franco R, Guarino MG, Barlattani A Jr, Bollero P. Experimental protocol of dental procedures in patients with hereditary angioedema: the role of anxiety and the use of nitrogen oxide. Oral Implantol (Rome) 2016; 9: 49-53.

20. Kirsch I, Deacon BJ, Huedo-Medina TB, Scoboria A, Moore TJ, Johnson BT. Initial severity and antidepressant benefits: a meta-analysis of data submitted to the food and drug administration. PLoS Med 2008; 5: e45.

21. Siebert T, Jurkovic R, Statelova D, Strecha J. Immediate implant placement in a patient with osteoporosis undergoing bisphosphonate therapy: 1-year preliminary prospective study. J Oral Implantol 2006; 41: 360-365.

22. Tai V, Leung W, Grey A, Reid IR, Bolland MJ. Calcium in-take and bone mineral density: systematic review and meta-analysis. BMJ 2006; 351: 4183

23. Zahid TM, Wang BY, Cohen RE. Influence of bisphosphonates on alveolar bone loss around osseointegrated implants. J Oral Implantol 2011; 37: 335-346.

24. Bollero P, Franco R, Cecchetti F, et al. Oral health and implant therapy in Parkinson's patients: review. Oral Implantol (Rome) 2017; 10: 105-111.

25. Memon S, Weltman RL, Katancik JA. Oral bisphosphonates: early endosseous dental implant success and crestal bone changes. A retrospective study. Int J Oral Maxillofac Implants 2012; 27: 1216-1222.

26. Diem SJ, Blackwell TL, Stone KL, Yaffe K, Haney EM, Bliziotes MM Use of antidepressants and rates of hip bone loss in older women the study of osteoporotic fractures. Arch Intern Med 2007; 167: 1240-1245.

27. Misch CE, Perel ML, Wang HL, Sammartino G, Galindo-Moreno P Trisi P. Implant success, survival, and failure: the International Congress of Oral Implantologists (ICOI) pisa consensus conference. Implant Dent 2008; 17: 5-15.

28. Tsapakis E, Gamie Z, Tran G, Adshead S, Lampard A, Mantalaris A The adverse skeletal effects of selective serotonin reuptake inhibitors. Eur Psychiatry 2012; 27: 156-169.

29. Wong M, Eulenberger J, Schenk R, Hunziker E. Effect of surface topology on the osseointegration of implant materials in trabecular bone. J Biomed Mater Res 1995; 29: 1567-1575. 
